\title{
A New Approach for Solving Boundary Value Problem in Partial Differential Equation Arising in Financial Market
}

\author{
Fadugba Sunday Emmanuel ${ }^{1}$, Emeka Helen Oluyemisi ${ }^{2}$ \\ ${ }^{1}$ Department of Mathematical Sciences, Ekiti State University, Ado Ekiti, Nigeria \\ ${ }^{2}$ Department of Mathematical and Physical Sciences, Afe Babalola University, Ado Ekiti, Nigeria \\ Email: "emmasfad2006@yahoo.com,dutireyemi@gmail.com
}

Received 1 April 2016; accepted 23 May 2016; published 26 May 2016

Copyright (C) 2016 by authors and Scientific Research Publishing Inc.

This work is licensed under the Creative Commons Attribution International License (CC BY). http://creativecommons.org/licenses/by/4.0/

c) (i) Open Access

\begin{abstract}
In this paper, we present a new approach for solving boundary value problem in partial differential equation arising in financial market by means of the Laplace transform. The result shows that the Laplace transform for the price of the European call option which pays dividend yield reduces to the Black-Scholes-Merton model.
\end{abstract}

\section{Keywords}

Black-Scholes-Merton Model, Boundary Value Problem, European Call Option, Financial Market, Laplace Transform

\section{Introduction}

An option is a contract that gives the right (not an obligation) to its holder to buy or sell some amount of the underlying asset at a future date for a prescribed price. The underlying assets include stocks, stock indices, debt instruments, commodities and foreign currency. A call option gives its holder the right to buy the underlying asset, whereas a put option gives its holder the right to sell. Vanilla options are actively traded on organized exchanges. They are also subject to certain regularity and standardization conditions. Vanilla options can be classified as American options and European options. An American option gives a financial agent the right, but not obligation to buy or to sell the underlying assets on or prior to the expiry date at the specified price called the exercise price. European option is an option that can be exercised only at the expiry date with linear payoff. European option comes in two forms namely European call and put options.

${ }^{*}$ Corresponding author.

How to cite this paper: Emmanuel, F.S. and Oluyemisi, E.H. (2016) A New Approach for Solving Boundary Value Problem in Partial Differential Equation Arising in Financial Market. Applied Mathematics, 7, 840-851.

http://dx.doi.org/10.4236/am.2016.79075 
A European call option is an option that can be exercised only at expiry and has a linear payoff given by the difference between underlying asset price at maturity and the exercise price. The payoff for the European call option is given by

$$
C\left(S_{T}, T\right)=\left(S_{T}-K\right)^{+} \text {. }
$$

A European put option is an option that can be exercised only at expiry and has a linear payoff given by the difference between the exercise price and underlying asset price at maturity. The payoff for the European put option is given by

$$
P\left(S_{T}, T\right)=\left(K-S_{T}\right)^{+} \text {. }
$$

The revolution on derivative securities both in exchange markets and in academic communities began in the early 1970's. How to rationally price an option was not clear until 1973, when Black and Scholes published their seminal work on option pricing in which they described a mathematical frame work for finding the fair price of a European option (see [1]).

Moreover, in the same year, [2] extended the Black-Scholes model in several important ways. Since its invention, the Black-Scholes formula has been widely used by traders to determine the price of an option. However this famous formula has been questioned after the 1987 crash.

One of the main concerns about financial options is what the exact values of options are. For the simplest model in the case of constant coefficients, an exact pricing formula was derived by Black and Scholes, known as the Black-Scholes formula. However, in the general case of time and space dependent coefficients the exact pricing formula is not yet established, and thus numerical solutions have been used (see [3]).

There are many exhaustive texts and literatures in this subject area such as [4]-[10], just to mention a few.

In this paper, we present a new approach for solving boundary value problem in partial differential equation arising in financial market via the Laplace transform. The rest of the paper is organized as follows: Section 2 presents the Black-Scholes-Merton partial differential equation for the price of European call option which pays a dividend yield. In Section 3, we consider the Laplace transform and some of its fundamental properties. Section 4 presents the Laplace transform for solving boundary value problem in partial differential equation arising in financial market. We also show that our result reduces to Black-Scholes-Merton like formula. Section 5 concludes the paper.

\section{The Black-Scholes-Merton Partial Differential Equation}

We consider a market where the underlying asset price $S_{t}, 0 \leq t \leq T$ is governed by the stochastic differential equation of the form

$$
\mathrm{d} S_{t}=(r-d) S_{t} \mathrm{~d} t+\sigma S_{t} \mathrm{~d} W_{t}, 0<S_{t}<\infty
$$

where $\sigma$ is the volatility, $r$ is the riskless interest rate, $d$ is the dividend yield and $W_{t}$ is a one-dimensional Wiener process. Standard arbitrage arguments show that any derivative $u\left(S_{t}, t\right)$ written on $S_{t}$ must satisfy the partial differential equation of the form

$$
\frac{\partial u\left(S_{t}, t\right)}{\partial t}+(r-d) S_{t} \frac{\partial u\left(S_{t}, t\right)}{\partial S_{t}}+\frac{1}{2} \sigma^{2} S_{t}^{2} \frac{\partial^{2} u\left(S_{t}, t\right)}{\partial S_{t}^{2}}=r u\left(S_{t}, t\right) .
$$

Setting $u\left(S_{t}, t\right)=C\left(S_{t}, t\right)$ in (4), then we have the Black-Scholes-Merton partial differential equation for the price of European call option given by

$$
\frac{\partial C\left(S_{t}, t\right)}{\partial t}+(r-d) S_{t} \frac{\partial C\left(S_{t}, t\right)}{\partial S_{t}}+\frac{1}{2} \sigma^{2} S_{t}^{2} \frac{\partial^{2} C\left(S_{t}, t\right)}{\partial S_{t}^{2}}=r C\left(S_{t}, t\right)
$$

with boundary conditions

$$
\begin{gathered}
C\left(S_{t}, t\right) \rightarrow \infty \text { as } S_{t} \rightarrow \infty \text { on }[0, T) \\
C\left(S_{t}, t\right) \rightarrow 0 \text { as } S_{t} \rightarrow 0 \text { on }[0, T)
\end{gathered}
$$


and final time condition given by

$$
C\left(S_{T}, T\right)=\left(S_{T}-K\right)^{+}=f\left(S_{T}\right) \text { on }[0, \infty) .
$$

Equation (7) states that the option is worthless when the stock price is zero.

\section{The Laplace Transform and Its Fundamental Properties}

Let $h(x)$ be a piece-wise continuous function on every closed interval $\{x \in[a, b] \subset x \in[0, \infty)\}$ there exists $h:\{x \in[0, \infty) \rightarrow \mathbb{R}\}, h: x \rightarrow h(x)$ such that $w \in \mathbb{R}$ and $w_{0} \in \mathbb{R}$. Then $L_{w}(h(x))=H(w)$, for $w \in \mathbb{R}$ is called the Laplace transform of $h(x)$ and is defined as

$$
L_{w}(h(x))=H(w)=\int_{0}^{\infty} h(x) \mathrm{e}^{-w x} \mathrm{~d} x, \forall w \in \mathbb{R}, w>w_{0}
$$

whenever the integral exists. Conversely, the inverse Laplace transform of $H(w)$ is defined as

$$
L_{w}^{-1}(H(w))=h(x)=\frac{1}{2 \pi i} \int_{c-i \infty}^{c+i \infty} H(w) \mathrm{e}^{w x} \mathrm{~d} w, c>w_{0} .
$$

Let $h(x)$ be a piece-wise continuous with the Laplace transform $L_{w}(h(x))$. The fundamental properties of the Laplace transform hold.

1) Linearity of the Laplace Transform

$$
L_{w}(a h(x))+L_{w}(b g(x))=a H(w)+b G(w)=\int_{0}^{\infty}(a h(x)+b g(x)) \mathrm{e}^{-w x} \mathrm{~d} x .
$$

Equation (11) is intermediate from the definition and the linearity of the definite integral.

2) Scaling Property

$$
L_{w}(a x)=\int_{0}^{\infty} h(a x) \mathrm{e}^{-w x} \mathrm{~d} x=\frac{1}{a} H\left(\frac{w}{a}\right), a>0
$$

3) Shifting Property

$$
L_{w}\left(\mathrm{e}^{a x}\right)=\int_{0}^{\infty} \mathrm{e}^{a x} \mathrm{e}^{-w x} \mathrm{~d} x=H(w-a)
$$

4) Commutativity Property

The Laplace transform is commutative. i.e.

$$
\begin{aligned}
L_{w}(g(x)) * L_{w}(h(x)) & =G(w) * H(w)=\int_{0}^{x} g(x-t) h(t) \mathrm{d} t \\
& =\int_{0}^{x} h(x-t) g(t) \mathrm{d} t=H(w) * G(w)=L_{w}(h(x)) * L_{w}(g(x))
\end{aligned}
$$

5) The Laplace Transforms on Differentiation

Let $h(x)$, for $x>0$ be a differentiable function with the derivative $\frac{\mathrm{d} h(x)}{\mathrm{d} x}$ being continuous. Suppose that there exist constant $M$ and $X$ such that $|h(x)| \leq M \mathrm{e}^{\alpha x}, \forall x \geq X$, then

$$
L_{w}(h(x))=H(w)=\int_{0}^{\infty} \frac{\mathrm{d} h(x)}{\mathrm{d} x} \mathrm{e}^{-w x} \mathrm{~d} x=\lim _{b \rightarrow \infty} \int_{0}^{b} \frac{\mathrm{d} h(x)}{\mathrm{d} x} \mathrm{e}^{-w x} \mathrm{~d} x=w H(0)-h(0) .
$$

Note that the condition

$$
|h(x)| \leq M \mathrm{e}^{\alpha x}, \forall x \leq X \Rightarrow \lim _{b \rightarrow \infty} h(b) \mathrm{e}^{-w b}=0, \text { for } w>\alpha .
$$

6) Convolution Property 


\section{Theorem 1: Convolution Theorem}

Let $L_{w}(g(x))$ and $L_{w}(h(x))$ denote the Laplace transforms of $g(x)$ and $h(x)$, respectively. Then the product given by $L_{w}(f(x))=L_{w}(g(x) h(x))$ is the Laplace transform of the convolution of $g(x)$ and $h(x)$ is denoted by $f(x)=\left(g^{*} h\right)(x)$ and the integral representation

$$
\left.\begin{array}{l}
f(x)=(g * h)(x)=\int_{0}^{x} g(x) h(x-s) \mathrm{d} s \\
f(x)=(h * g)(x)=\int_{0}^{x} h(x) g(x-s) \mathrm{d} s
\end{array}\right\}
$$

We present some of the results on the existence and uniqueness of the Laplace transform below.

\section{Theorem 2: Existence of Laplace Transform}

Let $h(x)$ be a piecewise continuous function on $[0, R]$ (for every $R>0$ ) and have an exponential order at infinity with $|h(x)| \leq M \mathrm{e}^{a x}$. Then, the Laplace transform $L_{w}(h(x))$ is defined for $w>\beta$, i.e.

$$
\{w>\beta\} \subset \text { Domain }\left\{L_{w}(h(x))\right\} .
$$

Theorem 3: Uniqueness of Laplace Transform Let $g(x)$ and $h(x)$ be two piecewise continuous functions with an exponential order at infinity. Assume that $L_{w}(g(x))=L_{w}(h(x))$, then $g(x)=h(x), x \in[0, P]$, for every $P>0$, except may be for a finite set of points.

\section{Relation to the Mellin and the Fourier Transformations}

Laplace transformation is closely related to an extended form of other popular transforms, particularly Mellin and Fourier. Both can be obtained through a change of variables. By setting

The Laplace transform (9) yields

$$
x=\mathrm{e}^{-z}, \mathrm{~d} x=-\mathrm{e}^{-z} \mathrm{~d} z .
$$

$$
M\left(h\left(\mathrm{e}^{-z}\right), w\right)=F\left(h\left(\mathrm{e}^{-z}\right),-i w\right)=L_{w}(h(x), w)
$$

where $M(),. F($.$) and L_{w}($.$) denote the Mellin transform, the Fourier transform and the Laplace transform re-$ spectively

\section{Laplace Transform for Solving Boundary Value Problems in Partial Differential Equation Arising in Financial Market}

By change of variables $\tau=T-t$, (5) becomes

$$
-\frac{\partial C\left(S_{\tau}, \tau\right)}{\partial t}+(r-d) S_{\tau} \frac{\partial C\left(S_{\tau}, \tau\right)}{\partial S_{\tau}}+\frac{1}{2} \sigma^{2} S_{\tau}^{2} \frac{\partial^{2} C\left(S_{\tau}, \tau\right)}{\partial S_{\tau}^{2}}=r C\left(S_{\tau}, \tau\right)
$$

with boundary conditions

$$
\begin{gathered}
C\left(S_{\tau}, \tau\right) \rightarrow \infty \text { as } S_{\tau} \rightarrow \infty \text { on }[0, T) \\
C\left(S_{\tau}, \tau\right) \rightarrow 0 \text { as } S_{\tau} \rightarrow 0 \text { on }[0, T)
\end{gathered}
$$

and final time condition given by

$$
C\left(S_{\tau}, \tau\right)=\left(S_{\tau}-K\right)^{+} \text {on }[0, \infty) .
$$

Let the Laplace transform for the price of the European call option be defined as

$$
L_{w}\left(C\left(S_{\tau}, \tau\right)\right)=f\left(S_{\tau}\right)
$$

and the inverse Laplace transform for the price of the European call option be given by

$$
L_{w}^{-1}\left(f\left(S_{\tau}\right)\right)=C\left(S_{\tau}, \tau\right)
$$


where $L_{w}$ is the Laplace operator and $f\left(S_{\tau}\right)$ is the Laplace transform with parameter $w$.

Taking the Laplace transform of (5) using (24) we have that

$$
L_{w}\left(-\frac{\partial C\left(S_{\tau}, \tau\right)}{\partial \tau}+(r-d) S_{\tau} \frac{\partial C\left(S_{\tau}, \tau\right)}{\partial S_{\tau}}+\frac{1}{2} \sigma^{2} S_{\tau}^{2} \frac{\partial^{2} C\left(S_{\tau}, \tau\right)}{\partial S_{\tau}^{2}}\right)=L_{w}\left(r C\left(S_{t}, \tau\right)\right)
$$

where

$$
\begin{aligned}
& L_{w}\left(-\frac{\partial C\left(S_{\tau}, \tau\right)}{\partial \tau}\right)=-\left(w f\left(S_{\tau}\right)-C(0, \tau)\right)=-w f\left(S_{\tau}\right)+\left(S_{\tau}-K\right)^{+} \\
& L_{w}\left((r-q) S_{\tau} \frac{\partial C\left(S_{\tau}, \tau\right)}{\partial S_{\tau}}\right)=(r-d) S_{\tau} \frac{\partial f\left(S_{\tau}\right)}{\partial S_{\tau}} \\
& L_{w}\left(\frac{1}{2} \sigma^{2} S_{\tau}^{2} \frac{\partial^{2} C\left(S_{\tau}, \tau\right)}{\partial S_{\tau}^{2}}\right)=\frac{1}{2} \sigma^{2} S_{\tau}^{2} \frac{\partial^{2} f\left(S_{\tau}\right)}{\partial S_{\tau}^{2}} \\
& L_{w}\left(r C\left(S_{\tau}, \tau\right)\right)=r f\left(S_{\tau}\right) .
\end{aligned}
$$

Substituting (27), (28), (29) and (30) into (26) yields

$$
-w f\left(S_{\tau}\right)+\left(S_{\tau}-K\right)^{+}+(r-d) S_{\tau} \frac{\partial f\left(S_{\tau}\right)}{\partial S_{\tau}}+\frac{1}{2} \sigma^{2} S_{\tau}^{2} \frac{\partial^{2} f\left(S_{\tau}\right)}{\partial S_{\tau}^{2}}=r f\left(S_{\tau}\right) .
$$

Simplifying further and rearranging terms in (31) we have that

$$
\frac{1}{2} \sigma^{2} S_{\tau}^{2} \frac{\partial^{2} f\left(S_{\tau}\right)}{\partial S_{\tau}^{2}}+(r-d) S_{\tau} \frac{\partial f\left(S_{\tau}\right)}{\partial S_{\tau}}-(r+w) f\left(S_{\tau}\right)=-\left(S_{\tau}-K\right)^{+}
$$

We consider the following two cases as follows.

\section{CASE I}

For $K \leq S_{\tau}$, (32) becomes

$$
\frac{1}{2} \sigma^{2} S_{\tau}^{2} \frac{\partial^{2} f\left(S_{\tau}\right)}{\partial S_{\tau}^{2}}+(r-d) S_{\tau} \frac{\partial f\left(S_{\tau}\right)}{\partial S_{\tau}}-(r+w) f\left(S_{\tau}\right)=-\left(S_{\tau}-K\right) .
$$

The general solution to (33) can be obtained as

$$
f_{1}\left(S_{\tau}\right)=f_{c 1}\left(S_{\tau}\right)+f_{p 1}\left(S_{\tau}\right)
$$

where $f_{c 1}\left(S_{\tau}\right)$ and $f_{p 1}\left(S_{\tau}\right)$ are the complementary solution to the homogeneous part of (33) which is of the form

$$
\frac{1}{2} \sigma^{2} S_{\tau}^{2} \frac{\partial^{2} f\left(S_{\tau}\right)}{\partial S_{\tau}^{2}}+(r-d) S_{\tau} \frac{\partial f\left(S_{\tau}\right)}{\partial S_{\tau}}-(r+w) f\left(S_{\tau}\right)=0
$$

and the particular solution respectively.

We assume that the solution to (33) is of the form

$$
f\left(S_{\tau}\right)=B S_{\tau}^{\alpha}
$$

The first and the second derivatives of (36) are obtained as

$$
\frac{\partial f\left(S_{\tau}\right)}{\partial S_{\tau}}=\alpha B S_{\tau}^{\alpha-1}
$$

and

$$
\frac{\partial^{2} f\left(S_{\tau}\right)}{\partial S_{\tau}^{2}}=\alpha(\alpha-1) B S_{\tau}^{\alpha-2} .
$$

Substituting (36), (37) and (38) into (35), and simplifying further, we have that 


$$
B S^{\alpha}\left[\frac{1}{2} \sigma^{2} \alpha^{2}+\left(r-d-\frac{1}{2} \sigma^{2}\right) \alpha-(r+w)\right]=0 .
$$

Solving (39), we obtain the following roots

$$
\begin{aligned}
& \alpha_{1}=\frac{-\left(r-d-\frac{1}{2} \sigma^{2}\right)+\sqrt{\left(r-d-\frac{1}{2} \sigma^{2}\right)^{2}+2 \sigma^{2}(r+w)}}{\sigma^{2}} \\
& \alpha_{2}=\frac{-\left(r-d-\frac{1}{2} \sigma^{2}\right)-\sqrt{\left(r-d-\frac{1}{2} \sigma^{2}\right)^{2}+2 \sigma^{2}(r+w)}}{\sigma^{2}}
\end{aligned}
$$

Hence the complementary solution to the homogeneous part of (33) is obtained as

$$
f_{c 1}\left(S_{\tau}\right)=B_{1} S_{\tau}^{\alpha_{1}}+B_{2} S_{\tau}^{\alpha_{2}}, \alpha_{2} \leq 0 \leq \alpha_{1}
$$

where $\alpha_{1}$ and $\alpha_{2}$ are given by (40).

For the particular solution of (33), we assume that

$$
f_{1 p}\left(S_{\tau}\right)=j S_{\tau}+l \Rightarrow \frac{\partial f_{1 p}\left(S_{\tau}\right)}{\partial S_{\tau}}=j \text { and } \frac{\partial^{2} f_{1 p}\left(S_{\tau}\right)}{\partial S_{\tau}^{2}}=0 .
$$

Using (42) and (33), and equating the coefficients of terms, we obtain

$$
\begin{aligned}
& j=\frac{1}{(w+d)} \\
& l=\frac{-K}{(r+w)} .
\end{aligned}
$$

Substituting (43) and (44) into the particular solution, we have that

$$
f_{1 p}\left(S_{\tau}\right)=\frac{S_{\tau}}{(w+d)}-\frac{K}{(r+w)} .
$$

Substituting (41) and (45) into (34)

$$
f_{1}\left(S_{\tau}\right)=B_{1} S_{\tau}^{\alpha_{1}}+B_{2} S_{\tau}^{\alpha_{2}}+\frac{S_{\tau}}{(w+d)}-\frac{K}{(r+w)}, \alpha_{2} \leq 0 \leq \alpha_{1} .
$$

Equation (46) is the general solution to (33) for $K \leq S_{\tau}$.

CASE II

For $K \geq S_{\tau}$, (33) becomes

$$
\frac{1}{2} \sigma^{2} S_{\tau}^{2} \frac{\partial^{2} f\left(S_{\tau}\right)}{\partial S_{\tau}^{2}}+(r-d) S_{\tau} \frac{\partial f\left(S_{\tau}\right)}{\partial S_{\tau}}-(r+w) f\left(S_{\tau}\right)=0 .
$$

Following the above procedures, the general solution to the last equation is obtained as

$$
f_{2}\left(S_{\tau}\right)=B_{1} S_{\tau}^{\alpha_{1}}+B_{2} S_{\tau}^{\alpha_{2}}, \alpha_{2} \leq 0 \leq \alpha_{1}
$$

with

$$
\alpha_{1}=\frac{-\left(r-d-\frac{1}{2} \sigma^{2}\right)+\sqrt{\left(r-d-\frac{1}{2} \sigma^{2}\right)^{2}+2 \sigma^{2}(r+w)}}{\sigma^{2}}
$$


Equation (47) coincides with the complementary solution of (33) given by (41).

In the case of $K \leq S_{\tau}$, we set $B_{1}=0$ to ensure the boundedness of the derivative $\frac{\partial f\left(S_{\tau}\right)}{\partial S_{\tau}}$. In the case of $K \geq S_{\tau}, B_{2}=0$ to ensure that the option's value approaches zero as the stock price goes to zero. The solutions for the two cases equations (46) and (47) become

$$
\begin{gathered}
f_{1}\left(S_{\tau}\right)=B_{2} S_{\tau}^{\alpha_{2}}+\frac{S_{\tau}}{(w+d)}-\frac{K}{(r+w)}, \alpha_{2} \leq 0 \leq \alpha_{1} \\
f_{2}\left(S_{\tau}\right)=B_{1} S_{\tau}^{\alpha_{1}}, \alpha_{2} \leq 0 \leq \alpha_{1} .
\end{gathered}
$$

We want the option pricing function to be continuous and differentiable at the transition point $K=S_{\tau}$, Therefore, the values of the function and their first derivatives from (48) and (49) must equal to each other. These conditions can be used to solve for $B_{1}$ and $B_{2}$. The function values and derivatives at $K=S_{\tau}$ from (48) and (49) are given by

$$
\begin{gathered}
\left.f_{1}\left(S_{\tau}\right)\right|_{K}=B_{2} K^{\alpha_{2}}+\frac{K}{(d+w)}-\frac{K}{(r+w)} \\
\left.\frac{\partial f\left(S_{\tau}\right)}{\partial S_{\tau}}\right|_{K}=B_{2} \alpha_{2} K^{\alpha_{2}-1}+\frac{1}{(d+w)} \\
\left.f_{2}\left(S_{\tau}, \tau\right)\right|_{K}=B_{1} K^{\alpha_{1}} \\
\left.\frac{\partial f\left(S_{\tau}\right)}{\partial S_{\tau}}\right|_{K}=B_{1} \alpha_{1} K^{\alpha_{1}-1} .
\end{gathered}
$$

Setting (50) $=(52)$ and $(51)=(53)$ and solving further, we obtain

$$
B_{1}=\left[\frac{\alpha_{2}}{(r+w)}-\frac{\left(\alpha_{2}-1\right)}{(d+w)}\right] \frac{1}{K^{\alpha_{1}-1}\left(\alpha_{1}-\alpha_{2}\right)}
$$

and

$$
B_{2}=\left[\frac{\alpha_{1}}{(r+w)}-\frac{\left(\alpha_{1}-1\right)}{(d+w)}\right] \frac{1}{K^{\alpha_{2}-1}\left(\alpha_{1}-\alpha_{2}\right)} .
$$

Substituting (54) and (55) into (49) and (48), we have

$$
f_{1}\left(S_{\tau}\right)=\left[\frac{\alpha_{1}}{(r+w)}-\frac{\left(\alpha_{1}-1\right)}{(d+w)}\right] \frac{1}{K^{\alpha_{2}-1}\left(\alpha_{1}-\alpha_{2}\right)} S_{\tau}^{\alpha_{2}}+\frac{S_{\tau}}{(w+d)}-\frac{K}{(r+w)}, \alpha_{2} \leq 0 \leq \alpha_{1}
$$

and

$$
f_{2}\left(S_{\tau}\right)=\left[\frac{\alpha_{2}}{(r+w)}-\frac{\left(\alpha_{2}-1\right)}{(d+w)}\right] \frac{1}{K^{\alpha_{1}-1}\left(\alpha_{1}-\alpha_{2}\right)} S_{\tau}^{\alpha_{1}}, \alpha_{2} \leq 0 \leq \alpha_{1}
$$

respectively. Equations (56) and (57) can also be written as

$$
f\left(S_{\tau}\right)=\left\{\begin{array}{l}
{\left[\frac{\alpha_{1}}{(r+w)}-\frac{\left(\alpha_{1}-1\right)}{(d+w)}\right] \frac{1}{K^{\alpha_{2}-1}\left(\alpha_{1}-\alpha_{2}\right)} S_{\tau}^{\alpha_{2}}+\frac{S_{\tau}}{(w+d)}-\frac{K}{(r+w)}, \alpha_{2} \leq 0 \leq \alpha_{1}, \text { for } K \leq S_{\tau}} \\
{\left[\frac{\alpha_{2}}{(r+w)}-\frac{\left(\alpha_{2}-1\right)}{(d+w)}\right] \frac{1}{K^{\alpha_{1}-1}\left(\alpha_{1}-\alpha_{2}\right)} S_{\tau}^{\alpha_{1}}, \alpha_{2} \leq 0 \leq \alpha_{1}, \text { for } K \geq S_{\tau}}
\end{array}\right.
$$

Equation (58) is the Laplace transform of the price of European call option which pays a dividend yield.

\section{Theorem 4}


If $K \geq S_{\tau}, \alpha_{2} \leq 0 \leq \alpha_{1}$, then the Laplace transform of the price of European call option with dividend yield given by

$$
f\left(S_{\tau}\right)=f_{1}\left(S_{\tau}\right)=\left[\frac{\alpha_{2}}{(r+w)}-\frac{\left(\alpha_{2}-1\right)}{(d+w)}\right] \frac{1}{K^{\alpha_{1}-1}\left(\alpha_{1}-\alpha_{2}\right)} S_{\tau}^{\alpha_{1}}
$$

reduces to the Black-Scholes-Merton valuation formula

$$
C_{B S M}\left(S_{\tau}, \tau\right)=S_{\tau} \mathrm{e}^{-d \tau} N\left(d_{1}\right)-K \mathrm{e}^{-r \tau} N\left(d_{2}\right)
$$

with

$$
\left.\begin{array}{c}
d_{1}=\frac{\ln \left(\frac{S_{\tau}}{K}\right)+\left(r-d+\frac{\sigma^{2}}{2}\right) \tau}{\sigma \sqrt{\tau}} \\
d_{2}=\frac{\ln \left(\frac{S_{\tau}}{K}\right)+\left(r-d-\frac{\sigma^{2}}{2}\right) \tau}{\sigma \sqrt{\tau}}=d_{1}-\sigma \sqrt{\tau}
\end{array}\right\}
$$

by means of the Laplace transform of the form

$$
L_{w}\left[\mathrm{e}^{g \tau} N\left(\frac{m+b \tau}{c \sqrt{\tau}}\right)\right]=\frac{1}{2} \mathrm{e}^{-a k} \frac{\mathrm{e}^{-k \sqrt{w-g+a^{2}}}}{\sqrt{w-g+a^{2}}\left(a+\sqrt{w-g+a^{2}}\right)}
$$

where

$$
\begin{aligned}
& \alpha_{1}=\frac{-\left(r-d-\frac{1}{2} \sigma^{2}\right)+\sqrt{\left(r-d-\frac{1}{2} \sigma^{2}\right)^{2}+2 \sigma^{2}(r+w)}}{\sigma^{2}}, \\
& \alpha_{2}=\frac{-\left(r-d-\frac{1}{2} \sigma^{2}\right)-\sqrt{\left(r-d-\frac{1}{2} \sigma^{2}\right)^{2}+2 \sigma^{2}(r+w)}}{\sigma^{2}},
\end{aligned}
$$

$a=-\frac{b}{c \sqrt{2}}$ and $k=-\frac{m \sqrt{2}}{c}$ and $b, c, m$ and $g$ are arbitrary constants.

Proof: From (57) and (58a) we can write that

$$
f\left(S_{\tau}\right)=f_{2}\left(S_{\tau}\right)=\frac{\alpha_{2}}{(r+w)} \frac{1}{K^{\alpha_{1}-1}\left(\alpha_{1}-\alpha_{2}\right)} S_{\tau}^{\alpha_{1}}-\frac{\left(\alpha_{2}-1\right)}{(d+w)} \frac{1}{K^{\alpha_{1}-1}\left(\alpha_{1}-\alpha_{2}\right)} S_{\tau}^{\alpha_{1}} .
$$

Setting

$$
\begin{gathered}
A_{1}=\frac{\alpha_{2}}{(r+w)} \frac{1}{K^{\alpha_{1}-1}\left(\alpha_{1}-\alpha_{2}\right)} S_{\tau}^{\alpha_{1}} \\
A_{2}=-\frac{\left(\alpha_{2}-1\right)}{(d+w)} \frac{1}{K^{\alpha_{1}-1}\left(\alpha_{1}-\alpha_{2}\right)} S_{\tau}^{\alpha_{1}} .
\end{gathered}
$$

Therefore, (59) becomes

$$
f\left(S_{\tau}\right)=f_{2}\left(S_{\tau}\right)=A_{1}+A_{2}
$$

Let us first consider the term

$$
A_{1}=\frac{\alpha_{2}}{(r+w)} \frac{1}{K^{\alpha_{1}-1}\left(\alpha_{1}-\alpha_{2}\right)} S_{\tau}^{\alpha_{1}}=K\left(\frac{\alpha_{2}}{r+w}\right)\left(\frac{1}{\alpha_{1}-\alpha_{2}}\right)\left(\frac{S}{K}\right)^{\alpha_{1}} .
$$


Using the values of

$$
\alpha_{2}=\frac{-\left(r-d-\frac{1}{2} \sigma^{2}\right)-\sqrt{\left(r-d-\frac{1}{2} \sigma^{2}\right)^{2}+2 \sigma^{2}(r+w)}}{\sigma^{2}}
$$

and

$$
\left(\alpha_{1}-\alpha_{2}\right)=\frac{2\left(\sqrt{\left(r-d-\frac{1}{2} \sigma^{2}\right)^{2}+2 \sigma^{2}(r+w)}\right)}{\sigma^{2}} .
$$

Therefore (63) yields

$$
\left.\begin{array}{l}
A_{1}=-K\left(\frac{\left(r-d-\frac{1}{2} \sigma^{2}\right)+\sqrt{\left(r-d-\frac{1}{2} \sigma^{2}\right)^{2}+2 \sigma^{2}(r+w)}}{(r+w) \sigma^{2}}\right)\left(\frac{\left(\frac{S_{\tau}}{K}\right)^{\alpha_{1}}}{\sigma^{2}\left(2 \sqrt{\left(r-d-\frac{1}{2} \sigma^{2}\right)^{2}+2 \sigma^{2}(r+w)}\right)}\right) \\
\left.\quad=-K\left(\frac{\left(r-d-\frac{1}{2} \sigma^{2}\right)+\sqrt{\left(r-d-\frac{1}{2} \sigma^{2}\right)^{2}+2 \sigma^{2}(r+w)}}{(r+w) \sigma^{2}}\right)\left(\frac{\mathrm{e}^{\alpha_{1} \ln \left(\frac{s_{\tau}}{K}\right)}}{\sigma^{2}\left(2 \sqrt{\left(r-d-\frac{1}{2} \sigma^{2}\right)^{2}+2 \sigma^{2}(r+w)}\right.}\right)\right)
\end{array}\right)
$$

Thus,

$$
\begin{aligned}
& \left.A_{1}=-K\left[\frac{\frac{1}{2} \mathrm{e}^{\alpha_{1} \ln \left(\frac{S_{\tau}}{K}\right)}\left(-\left(r-d-\frac{1}{2} \sigma^{2}\right)^{2}+\left(r-d-\frac{1}{2} \sigma^{2}\right)+2 \sigma^{2}(r+w)\right)}{(r+w) \sqrt{\left(r-d-\frac{1}{2} \sigma^{2}\right)^{2}+2 \sigma^{2}(r+w)}\left[-\left(r-d-\frac{1}{2} \sigma^{2}\right)+\sqrt{\left(r-d-\frac{1}{2} \sigma^{2}\right)^{2}+2 \sigma^{2}(r+w)}\right.}\right]\right] \\
& =-K\left[\frac{\frac{1}{2} \mathrm{e}^{\alpha_{1} \ln \left(\frac{s_{\tau}}{K}\right)}}{\frac{1}{2 \sigma^{2}} \sqrt{\left(r-d-\frac{1}{2} \sigma^{2}\right)^{2}+2 \sigma^{2}(r+q)}\left[-\left(r-d-\frac{1}{2} \sigma^{2}\right)+\sqrt{\left(r-d-\frac{1}{2} \sigma^{2}\right)^{2}+2 \sigma^{2}(r+w)}\right]}\right]
\end{aligned}
$$

Substituting the value of $\alpha_{1}$ into (64) and simplifying further, we obtain

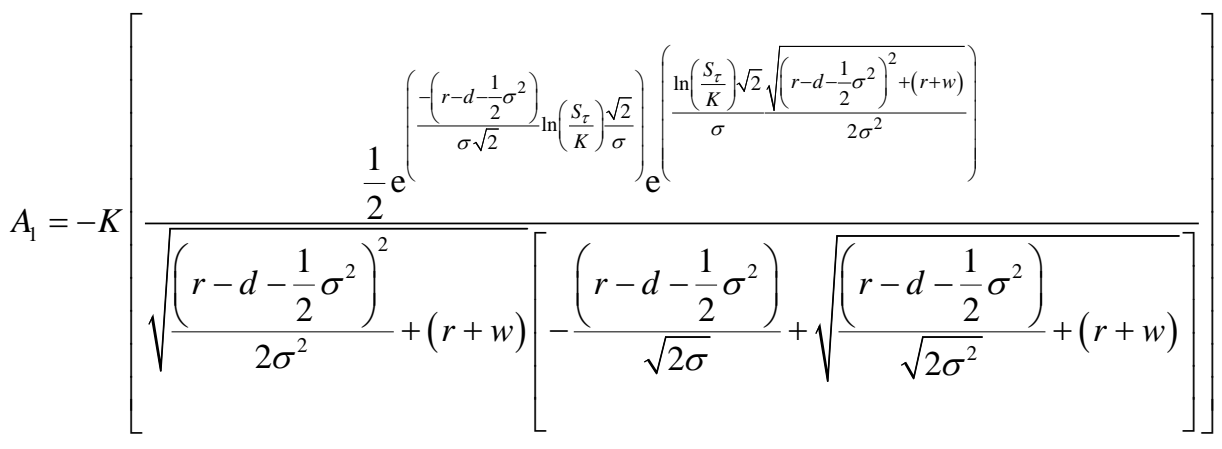


Comparing (65) with (58c), we have that

$$
\left.\begin{array}{l}
b=\left(r-d-\frac{1}{2} \sigma^{2}\right), c=\sigma, m=\ln \left(\frac{S_{\tau}}{K}\right), \\
g=-r, a=-\frac{b}{c \sqrt{2}}=-\frac{\left(r-d-\frac{1}{2} \sigma^{2}\right)}{\sigma \sqrt{2}}, \\
k=-\frac{m \sqrt{2}}{c}=-\frac{\ln \left(\frac{S_{\tau}}{K}\right) \sqrt{2}}{\sigma} .
\end{array}\right\}
$$

Taking the inverse Laplace transform of (65), we obtain

$$
\bar{A}_{1}=-K \mathrm{e}^{-r \tau} N\left(d_{1}-\sigma \sqrt{\tau}\right)=-K \mathrm{e}^{-r \tau} N\left(d_{2}\right)
$$

where

$$
d_{2}=\frac{\ln \left(\frac{S_{\tau}}{K}\right)+\left(r-d-\frac{1}{2} \sigma^{2}\right) \tau}{\sigma \sqrt{\tau}} .
$$

We also consider the term

$$
\begin{aligned}
A_{2} & =-\frac{\left(\alpha_{2}-1\right)}{(d+w)} \frac{1}{K^{\alpha_{1}-1}\left(\alpha_{1}-\alpha_{2}\right)} S_{\tau}^{\alpha_{1}} \\
& =-\frac{\left(\alpha_{2}-1\right)}{(d+w)}\left(\frac{S_{\tau}}{\left(\alpha_{1}-\alpha_{2}\right)}\right)\left(\frac{S_{\tau}}{K}\right)^{\alpha_{1}-1} .
\end{aligned}
$$

Substituting the values of $\alpha_{1}$ and $\alpha_{2}$ into (69) yields

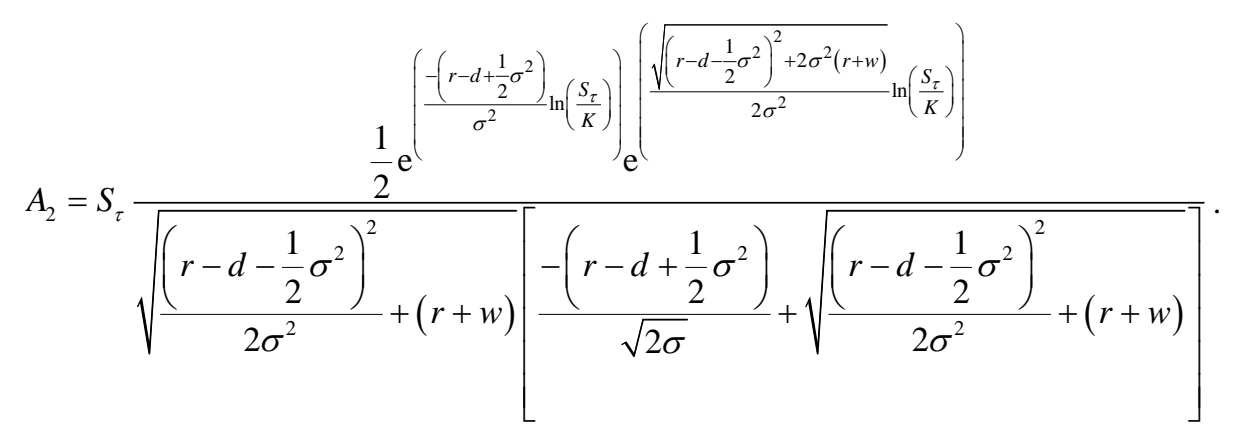

Simplifying (70) further, we obtain

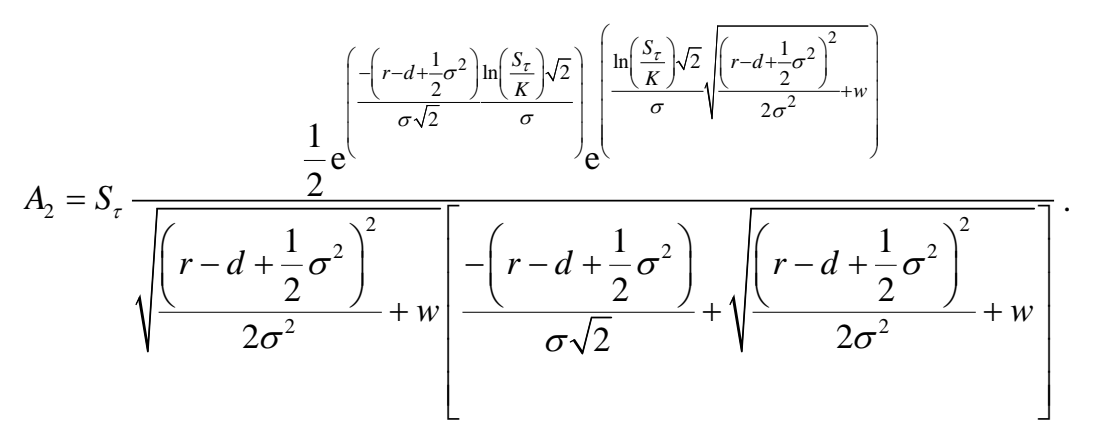

Once again we compare (71) with (58c), we deduce that 


$$
\left.\begin{array}{l}
b=\left(r-d+\frac{1}{2} \sigma^{2}\right), c=\sigma, m=\ln \left(\frac{S_{\tau}}{K}\right), \\
g=-d, a=-\frac{b}{c \sqrt{2}}=-\frac{\left(r-d+\frac{1}{2} \sigma^{2}\right)}{\sigma \sqrt{2}}, \\
k=-\frac{m \sqrt{2}}{c}=-\frac{\ln \left(\frac{S_{\tau}}{K}\right) \sqrt{2}}{\sigma}
\end{array}\right\}
$$

Taking the Laplace transform of (72), we have that

$$
\bar{A}_{2}=S_{\tau} \mathrm{e}^{-d \tau} N\left(d_{1}\right)
$$

where

$$
d_{1}=\frac{\ln \left(\frac{S_{\tau}}{K}\right)+\left(r-d+\frac{1}{2} \sigma^{2}\right) \tau}{\sigma \sqrt{\tau}} .
$$

The inverse Laplace transform of (62) is obtained as

$$
C\left(S_{\tau}, \tau\right)=C_{2}\left(S_{\tau}, \tau\right)=\bar{A}_{1}+\bar{A}_{2} .
$$

Substituting (67), (68), (73) and (74) into (75) yields

$$
\begin{aligned}
& C\left(S_{\tau}, \tau\right)=C_{2}\left(S_{\tau}, \tau\right)=S_{\tau} \mathrm{e}^{-d \tau} N\left(d_{1}\right)-K \mathrm{e}^{-r \tau} N\left(d_{2}\right)=C_{B S M}\left(S_{\tau}, \tau\right) \\
& d_{1}=\frac{\ln \left(\frac{S_{\tau}}{K}\right)+\left(r-d+\frac{1}{2} \sigma^{2}\right) \tau}{\sigma \sqrt{\tau}} \\
& d_{2}=\frac{\ln \left(\frac{S_{\tau}}{K}\right)+\left(r-d-\frac{1}{2} \sigma^{2}\right) \tau}{\sigma \sqrt{\tau}}=d_{1}-\sigma \sqrt{\tau}
\end{aligned}
$$

This completes the proof.

\section{Theorem 5}

If $K \leq S_{\tau}, \alpha_{2} \leq 0 \leq \alpha_{1}$, then the Laplace transform of the price of European call option with dividend yield given by

$$
C\left(S_{\tau}, \tau\right)=\left[\frac{\alpha_{1}}{(r+w)}-\frac{\left(\alpha_{1}-1\right)}{(d+w)}\right] \frac{1}{K^{\alpha_{2}-1}\left(\alpha_{1}-\alpha_{2}\right)} S_{\tau}^{\alpha_{2}}+\frac{S_{\tau}}{(w+d)}-\frac{K}{(r+w)}
$$

reduces to the Black-Scholes-Merton valuation formula given by

$$
C_{B S M}\left(S_{\tau}, \tau\right)=S_{\tau} \mathrm{e}^{-d \tau} N\left(d_{1}\right)-K \mathrm{e}^{-r \tau} N\left(d_{2}\right)
$$

with

$$
\left.\begin{array}{c}
d_{1}=\frac{\ln \left(\frac{S_{\tau}}{K}\right)+\left(r-d+\frac{\sigma^{2}}{2}\right) \tau}{\sigma \sqrt{\tau}} \\
d_{2}=\frac{\ln \left(\frac{S_{\tau}}{K}\right)+\left(r-d-\frac{\sigma^{2}}{2}\right) \tau}{\sigma \sqrt{\tau}}=d_{1}-\sigma \sqrt{\tau}
\end{array}\right\}
$$

by means of the Laplace transform given by 


$$
L_{w}\left[\mathrm{e}^{g \tau} N\left(\frac{m+b \tau}{c \sqrt{\tau}}\right)\right]=\frac{1}{2} \mathrm{e}^{-a k} \frac{\mathrm{e}^{-k \sqrt{w-g+a^{2}}}}{\sqrt{w-g+a^{2}}\left(a+\sqrt{w-g+a^{2}}\right)}
$$

where

$$
\alpha_{1}=\frac{-\left(r-d-\frac{1}{2} \sigma^{2}\right)+\sqrt{\left(r-d-\frac{1}{2} \sigma^{2}\right)^{2}+2 \sigma^{2}(r+w)}}{\sigma^{2}},
$$

$a=-\frac{b}{c \sqrt{2}}$ and $k=-\frac{m \sqrt{2}}{c}$ and $b, c, m$ and $g$ are arbitrary constants.

\section{Remark 1}

1) The proof of Theorem 5 follows from Theorem 4, since (56) and (57) have the same inverse Laplace transforms.

2 The above results show that the prices of European call option with dividend yield given by (56) and (57) coincide with the Black-Scholes-Merton model given by (58b) by means of (58c).

\section{Conclusion}

Finance is one of the fastest developing areas in the modern corporate and banking world. In this paper, we have considered the boundary value problem in partial differential equation arising in financial market. We used a new approach for solving the Black-Scholes-Merton partial differential equation for the price of European call option which pays a dividend yield by means of the Laplace transform. The same approach can be used for European put option with dividend paying stock. The results show that the Laplace transform for the price of the European call option with dividend paying stock coincides with the Black-Scholes-Merton model; it is very effective and is a good tool for solving partial differential equations arising in financial market and other areas such as engineering and applied sciences.

\section{References}

[1] Black, F. and Scholes, M. (1973) The Pricing of Options and Corporate Liabilities. Journal of Political Economy, 81, 637-654. http://dx.doi.org/10.1086/260062

[2] Merton, R.C. (1973) Theory of Rational Option Pricing. Bell Journal of Economics and Management Science, 4, 141183. http://dx.doi.org/10.2307/3003143

[3] Lee, H. and Sheen, D. (2009) Laplace Transformation Method for the Black-Scholes Equation. International Journal of Numerical Analysis and Modelling, 6, 642-658.

[4] Cohen, A.M. (2007) Numerical Methods for Laplace Transform Inversion. Springer, New York.

[5] Nwozo, C.R. and Fadugba, S.E. (2014) Performance Measure of Laplace Transforms for Pricing Path Dependent Options. International Journal of Pure and Applied Mathematics, 94, 175-197. http://dx.doi.org/10.12732/ijpam.v94i2.5

[6] Fadugba, S.E. and Ogunrinde, R.B. (2014) Black-Scholes Partial Differential Equation in the Mellin Transform Domain. International Journal of Scientific and Technology Research, 3, 200-206.

[7] Lee, R. (2003) Option Pricing by Transform Methods: Extension, Unification and Error Control. Stanford University, Working Paper.

[8] Petrella, G. and Kuo, S.G. (2004) Numerical Pricing of Discrete Barrier and Lookback Options via Laplace Transforms. Journal of Computational Finance, 8, 1-37.

[9] Zieneb, E. and Rokiah, R.A. (2013) Solving an Asian Options PDE via Laplace Transform. Science Asia, 39S, 67-69. http://dx.doi.org/10.2306/scienceasia1513-1874.2013.39S.067

[10] Shimko, D.C. (1992) Finance in Continuous Time. A Primer, Kolb Publishing. 